\title{
Um estudo sobre os possíveis contatos envolvendo alunos em salas de aulas
}

\section{A study about possible contacts involving students in classrooms}

DOI: $10.46814 /$ lajdv3n4-030

Recebimento dos originais: 01/05/2021

Aceitação para publicação: 31/06/2021

\section{Severino Horácio da Silva}

Unidade Acadêmica de Matemática. Universidade Federal de Campina Grande.

E-mail: horaciousp@gmail.com.

\section{Michelli Karinne Barros da Silva}

Unidade Acadêmica de Estatística. Universidade Federal de Campina Grande.

E-mail: michelli.karinne@gmail.com

\section{RESUMO}

No início da pandemia da COVID-19 as escolas brasileiras foram fechadas com o objetivo de conter a disseminação do vírus. Diante da discussão sobre a reabertura das escolas, neste trabalho, consideramos alguns cenários típicos de salas de aulas e através de um cálculo combinatório deduzimos o número de pessoas que cada aluno estaria exposto, bem como o número de possíveis contatos cruzados entre cada aluno e as pessoas expostas. Os cenários considerados foram ilustrados por figuras construídas no software de geometria dinâmica GeoGebra, onde as pessoas são representadas por vértices de polígonos e os contatos cruzados entre as pessoas pelas diagonais desses polígonos. Acreditamos que as informações referentes a contatos sejam relevantes, em momentos de pandemia, uma vez que os contatos potencializam o contágio.

Palavras-chave: Atividades escolares, COVID-19, Cálculo Combinatório, GeoGebra.

\begin{abstract}
At the beginning of the COVID-19 pandemic, Brazilian schools were closed in order to contain the spread of the virus. In view of the discussion about the reopening of schools, in this paper we consider some typical classroom scenarios and through a combinatorial calculation we deduce the number of people each student would be exposed to, as well as the number of possible cross-contacts between each student and the exposed people. The scenarios considered were illustrated by figures built in the dynamic geometry software GeoGebra, where people are represented by vertices of polygons and the cross-contacts between people by the diagonals of these polygons. We believe that information regarding contacts is relevant in times of pandemic, since contacts potentiate contagion.
\end{abstract}

Keywords: School Activities, COVID-19, Combinatorial Calculus, GeoGebra. 


\section{INTRODUÇÃO}

Atualmente o mundo está passando pela pandemia da COVID-19, causada pelo novo coronavírus denominado SARSCoV-2, sigla do inglês, Severe Acute Respiratory Syndrome Coronavírus 2 (que significa Síndrome Respiratória Aguda Severa causada pelo Coronavírus 2). A COVID-19 é uma doença recente e a comunidade científica ainda está aprendendo como ela afeta as crianças e qual o papel dessa na sua disseminação (KISHORE, 2020). No início da pandemia, a maioria dos relatos descrevia a população pediátrica como de baixo risco para COVID-19. Um estudo realizado na China com pacientes pediátricos com COVID-19 mostrou que 90\% dos pacientes eram assintomáticos, casos leves ou moderados, sugerindo que as infecções em crianças são menos graves do que em pacientes adultos (DONG et al. 2020). Já Kishore (2020) relata que as crianças podem correr um risco maior de contrair infecção se já apresentarem alguma comorbidade, como doenças respiratórias (asma, bronquite), doenças neurológicas, genéticas, metabólicas, doenças cardíacas congênitas ou em um estado imunocomprometido. No entanto, conforme descrito em Siegenfeld et al. (2020), inicialmente, foram cometidos dois equívocos: supor que as crianças raramente eram infectadas e que quando infectadas, raramente apresentariam um quadro grave da doença. Segundo os autores, um estudo de dados de rastreamento de contato revelou que crianças de 0-14 anos eram tão suscetíveis ao vírus quanto as pessoas com idades entre 15-64 anos. Além disso, Riollano-Cruz et al. (2020), relataram que em 27 de abril de 2020, The Pediatric Intensive Care Society of the United Kingdom lançou um alerta sobre o aumento do número de crianças com doença inflamatória multissistêmica, com características de doença que se sobrepõem à doença de Kawasaki (KD) e à síndrome do choque tóxico, muitas das quais tiveram resultado positivo para COVID-19. Em 4 de maio de 2020, o Departamento de Saúde do Estado de Nova York (NYSDOH) relatou 15 casos iniciais com sintomas semelhantes aos descritos e, em 17 de maio de 2020, 145 casos suspeitos foram notificados em hospitais do Estado de Nova York. Os primeiros 15 pacientes que apresentaram esta doença inflamatória multissistêmica foram estudados em Riollano-Cruz et al. (2020), onde relataram a presença de anticorpos para o SARSCoV-2 em todos os 15 pacientes estudados.

Segundo Beghi et al., (2020), o surto causado pelo SARS-CoV-2 apresenta manifestações neurológicas em grande parte dos indivíduos afetados, como distúrbios do sistema nervoso central e periférico, enquanto acidente vascular cerebral, ataxia, convulsões e depressão do nível de consciência são mais comuns em pacientes gravemente afetados. Pacientes com envolvimento do sistema nervoso, como sintomas manifestos nos estágios iniciais da infecção, podem ser diagnosticados incorretamente e podem disseminar o vírus inadvertidamente (FIANI, 2020).

Também há evidências crescentes sugerindo que indivíduos pré-sintomáticos têm altas cargas virais e são responsáveis por uma grande proporção da transmissão do SARSCoV-2. Apesar da 
letalidade da COVID-19 ser mais frequente em idosos e pessoas com comorbidade, as infecções das pessoas com comorbidades podem ser provocadas por indivíduos infectados que não apresentam sintomas da doença, já que estimativas recentes sugerem que 15-45\% de todas as infecções por SARSCoV-2 são assintomáticas (VERMUND e PITZER, 2020).

A disseminação de uma doença contagiosa depende fortemente do número de indivíduos suscetíveis (que ainda não contraíram a doença), do número de indivíduos infectados (que contraíram a doença e podem contagiar outras pessoas) e da taxa de contato entre os indivíduos suscetíveis e os infectados, veja por exemplo, (ALMEIDA et al., 2014; BATISTA e DA SLVA, 2020, CASTILHO, 2020; FARIAS, 2017; KERMARCK e MCKENDRICK, 1927 e NICOLA, 2020).

Denotando por $p$ a probabilidade de infecção para cada contato e por $n$ o número de vezes que uma pessoa infectada tem contato com outros indivíduos suscetíveis (em uma unidade de tempo) temos que a capacidade de um indivíduo infectado contagiar outros indivíduos será $n p$ ver (FARIAS, 2017). Portanto, quanto mais contatos maior a capacidade de disseminação da doença (de um vírus), por isso o distanciamento social é recomendado para diminuir a força do contágio de uma pandemia. Sendo assim, o Brasil e a maioria dos países do mundo adotaram políticas de distanciamento social para diminuir a velocidade de contágio e evitar que os sistemas de saúde entrassem em colapso.

Considerando que o ambiente escolar é um local típico de aglomerações e contatos entre as pessoas, as atividades escolares presenciais foram suspensas no início da pandemia. De acordo com Siegenfeld et al. (2020), tem havido opiniões distintas sobre o papel das escolas na propagação do SARSCoV-2. Algumas análises científicas confirmam o grande papel que as escolas desempenham na propagação do vírus, enquanto há outras que não sugerem evidências de grandes efeitos do fechamento de escolas. Ainda de acordo com os autores, os resultados divergentes podem ser devido a diferentes suposições de modelagem, bem como heterogeneidade nas sociedades estudadas. Por exemplo, se o vírus estiver bem contido, é menos provável que as escolas espalhem o vírus, como foi o caso da Dinamarca, que foi o primeiro país Europeu a reabrir as escolas de forma gradual e não teve um descontrole na disseminação do vírus. Vale ressaltar que a Dinamarca reabriu as escolas quando o número de novos casos e de óbitos pela COVID-19 estavam em queda e o país seguia com regras rígidas de distanciamento social, com fronteiras fechadas e sendo proibido aglomerações com mais de 10 pessoas. Além disso, os espaços físicos foram modificados, a quantidades de alunos por sala reduzida e os professores das séries iniciais conduzindo os alunos para ambientes como parquinhos e academias ao invés de ficarem trabalhando apenas na sala com a lousa (CAHAPAY, 2020). Alguns exemplos de escolas e creches que contribuíram substancialmente para a propagação do vírus foram: no Texas, em 6 de julho de 2020, havia 894 casos confirmados entre funcionários e 441 entre crianças de 883 creches do estado. Em contraste, o Texas relatou apenas 59 casos totais em 53 creches em 15 
de maio de 2020. Em Israel, que reduziu o número total de novos casos em todo o país para menos de 20 por dia em meados de maio, houve 130 casos de uma única escola até 3 de junho, apenas duas semanas após as escolas serem reabertas. Na Inglaterra, quase 20\% dos surtos de 15-21 de junho foram das escolas (para maiores detalhes ver (SIEGENFELD et al., 2020)).

De acordo com Ribeiro et al. (2020) as escolas funcionam como um meio pelo qual se semeia novas atitudes para a promoção da saúde e do bem-estar social. No entanto, num período pandêmico deve-se ter cautela, pois segundo Siegenfeld et al. (2020), escolas e creches causam eventos de superpropagação e que enquanto a transmissão do vírus na comunidade persiste, escolas e creches que reabrem ou permanecer aberto provavelmente causará eventos de disseminação adicional que irão dificultar os esforços de contenção.

Diante da polêmica do papel da escola na disseminação do SARSCoV-2, este trabalho propõe uma análise sobre os possíveis contatos de pessoas diferentes que uma criança estará exposta ao frequentar uma sala de aula. Essa análise foi motivada pelo trabalho coordenado pelo professor Alberto Aragón, da Universidade de Granada, na Espanha e divulgado no Jornal El País, de 17 de junho de 2020.

\section{ANÁLISE DE PROVÁVEIS CONTATOS CRUZADOS EM AMBIENTE ESCOLAR}

Para estudar os possíveis contatos cruzados em ambiente escolar vamos simular situações reais, considerando três cenários, com 10, 20 e 30 alunos na sala de aula.

\subsection{CENÁRIO 1}

Vamos supor uma sala de aula com 10 alunos e um professor, com cada um desses alunos morando apenas com seus pais e um irmão e o professor morando com seu cônjuge e mais dois filhos.

Na Figura 1, ilustramos a distribuição de 10 crianças nos vértices de um polígono regular de 10 lados e o professor no centro do polígono. Os contatos diretos entre as crianças são representados pelas arestas do polígono regular, as diagonais desse e os lados e diagonais de um dos quadriláteros externo ao polígono regular. Os contatos indiretos são representados pelas arestas e diagonais dos demais quadriláteros externos ao polígono. $O$ ponto $P$ denota o professor, o ponto $C_{j}$ denota a $j$-ésima criança e $M_{j}, P_{j}$ e $I_{j}$ denotam, respectivamente, a mãe, o pai e o irmão da criança $C_{j}$, com $j=1,2, \ldots, 10$ e $F P_{1}$ e $F P_{2}$ denotam os filhos do professor.

Com as hipóteses assumidas acima, em um único dia de aula, qualquer uma dessas pessoas estará exposta a 43 pessoas diferentes e existe a possibilidade de 121 contatos cruzados, conforme ilustração na Figura 1. Se pelo menos uma dessas pessoas estiver com o vírus SarsCov-2 (que provoca 
a COVID-19), o risco de contágio é muito grande porque a capacidade de um indivíduo infectado contagiar outros indivíduos será multiplicada pelo número de contatos.

Figura1: Ilustração dos possíveis contatos cruzados de uma criança numa sala com 20 alunos.

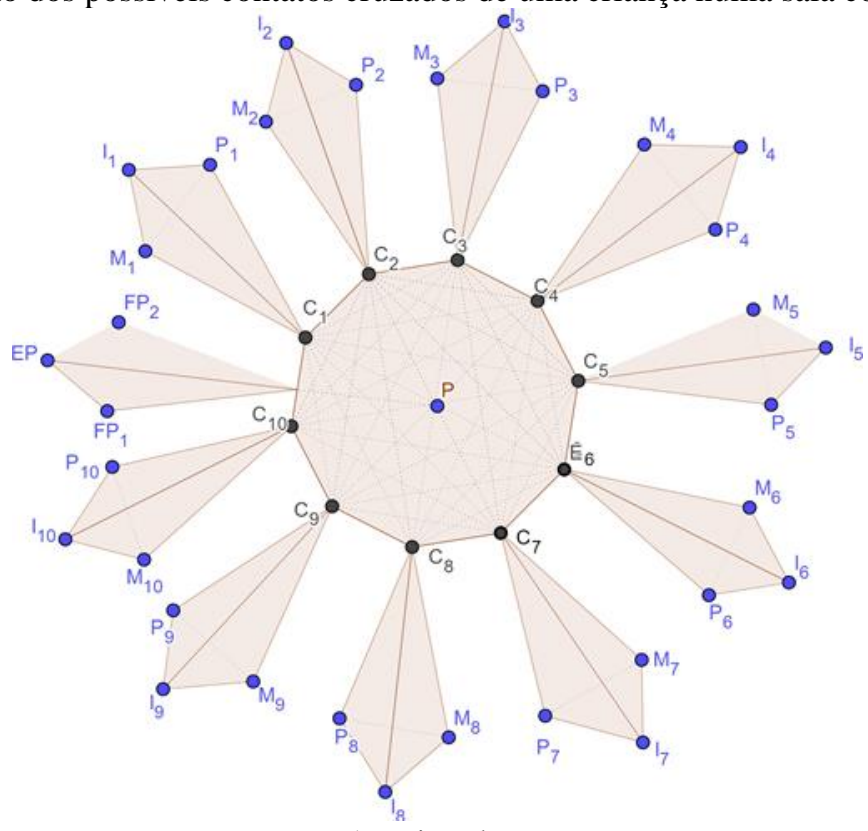

Fonte: Arquivo dos autores

O número de pessoas expostas, nesse caso, pode ser calculado por:

$$
N_{P E}=\left(n_{c}+1\right)+\left(n_{c}+1\right) \times 3,
$$

em que $N_{P E}$ representa o número de pessoas expostas e $n_{c}$ representa o número de crianças na sala.

Para o número de contatos cruzados podemos usar a seguinte fórmula:

$$
N_{c c}=\left(\begin{array}{c}
n_{c}+1 \\
2
\end{array}\right)+\left(n_{c}+1\right) \times\left(\begin{array}{l}
4 \\
2
\end{array}\right)
$$

em que $N_{c c}$ representa o número de contatos cruzados e $\left(\begin{array}{l}q \\ r\end{array}\right)$ representa o número de combinações de $q$ elementos tomados $r$ a $r$.

É válido salientar que na ilustração da Figura 1 está sendo considerado que a turma tem um único professor. Logo é uma situação típica do Ensino Infantil ou eventualmente do Ensino Fundamental Anos Iniciais. Além disso, foi assumido que os alunos e o professor não tiveram contato com nenhuma outra pessoa como porteiro, auxiliar de classe, acompanhante terapêutica, auxiliar de serviços gerais, professores de educação física, coordenador pedagógico, psicólogo ou outra pessoa fora da classe ou da sua residência, o que é uma situação não muito realística. 


\subsection{CENÁRIO 2}

Considerando uma sala de aula com 20 alunos que moram com seus pais e um único irmão e um professor (solteiro e sem filhos), num primeiro dia de aula, cada uma dessas crianças estará exposta a 80 pessoas diferentes e 330 contatos cruzados, conforme Figura 2. Se pelo menos uma dessas pessoas estiver com o vírus SarsCov-2 a capacidade de um indivíduo infectado contagiar outros indivíduos será muito maior do que na configuração anterior, já que passamos de 43 para 330 contatos cruzados.

Nesse caso, usando a mesma notação do Cenário 1, o número de pessoas expostas pode ser calculado por:

$$
N_{P E}=\left(n_{c}+1\right)+\left(n_{c} \times 3\right)
$$

e o número de contatos cruzados obtido por

$$
N_{c c}=\left(\begin{array}{c}
n_{c}+1 \\
2
\end{array}\right)+n_{c} \times\left(\begin{array}{l}
4 \\
2
\end{array}\right)
$$

Figura 2: Ilustração dos possíveis contatos cruzados de uma criança numa sala com 20 alunos

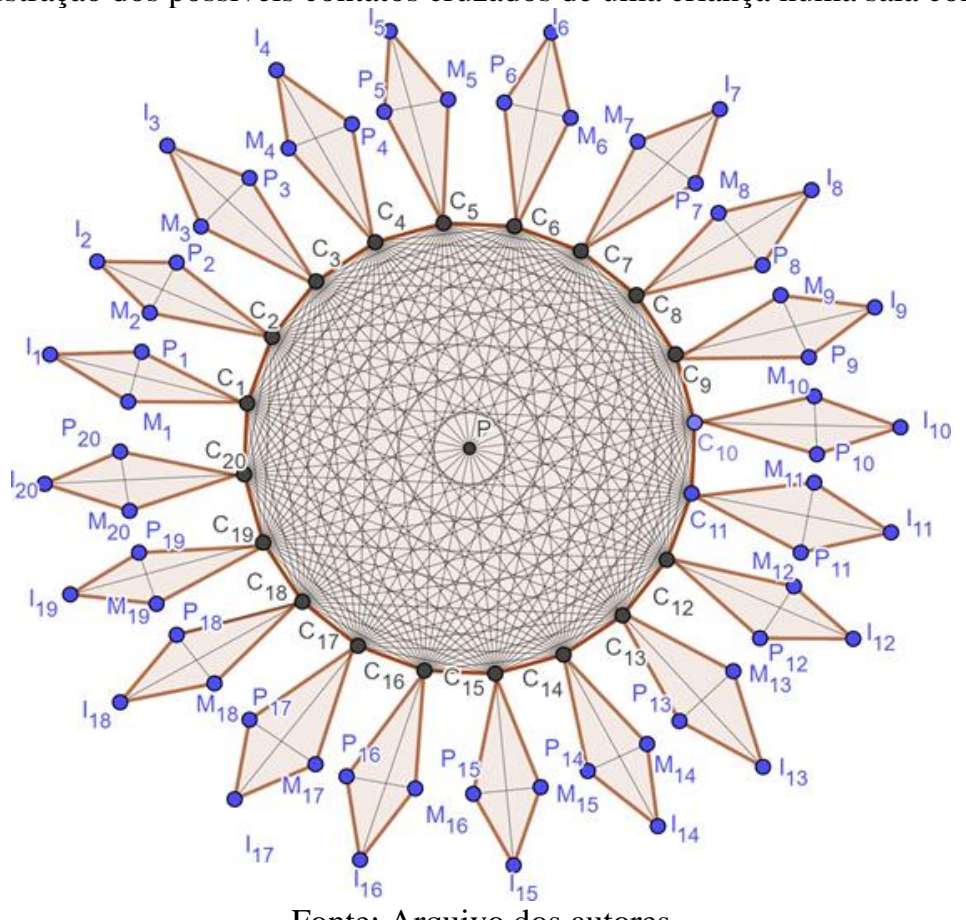

Fonte: Arquivo dos autores

\subsection{CENÁRIO 3}

Considerando uma sala de aula com 30 alunos e 10 professores (típico de uma turma do Ensino Fundamental II ou possivelmente do Ensino Médio) em que 15 alunos tem um irmão e os outros 15 
são filhos únicos e 5 professores tem dois filhos e 5 só tem um filho, teremos que cada pessoa do grupo estará exposto a 139 pessoas diferentes e o número de contatos cruzados pode chegar a 960.

Nesse caso, denotando por $n_{c}$ o número de crianças e por $n_{p}$ o número de professores, o número de pessoas expostas pode ser calculado por

$$
N_{P E}=\left(n_{c}+n_{p}\right)+\left(\frac{n_{c}}{2}+\frac{n_{p}}{2}\right) \times 3+\left(\frac{n_{c}}{2}+\frac{n_{p}}{2}\right) \times 2
$$

e o número de contatos cruzados obtido por

$$
N_{c c}=\left(\begin{array}{c}
n_{c+} n_{p} \\
2
\end{array}\right)+\left(\frac{n_{c}}{2}+\frac{n_{p}}{2}\right) \times\left(\begin{array}{l}
3 \\
2
\end{array}\right)+\left(\frac{n_{c}}{2}+\frac{n_{p}}{2}\right) \times\left(\begin{array}{l}
4 \\
2
\end{array}\right) .
$$

Para essa configuração, ilustramos na Figura 3, os contatos cruzados apenas das crianças e sua família o que corresponde a 570 contatos.

Figura 3: Ilustração dos possíveis contatos cruzados de uma criança numa sala com 30 alunos.

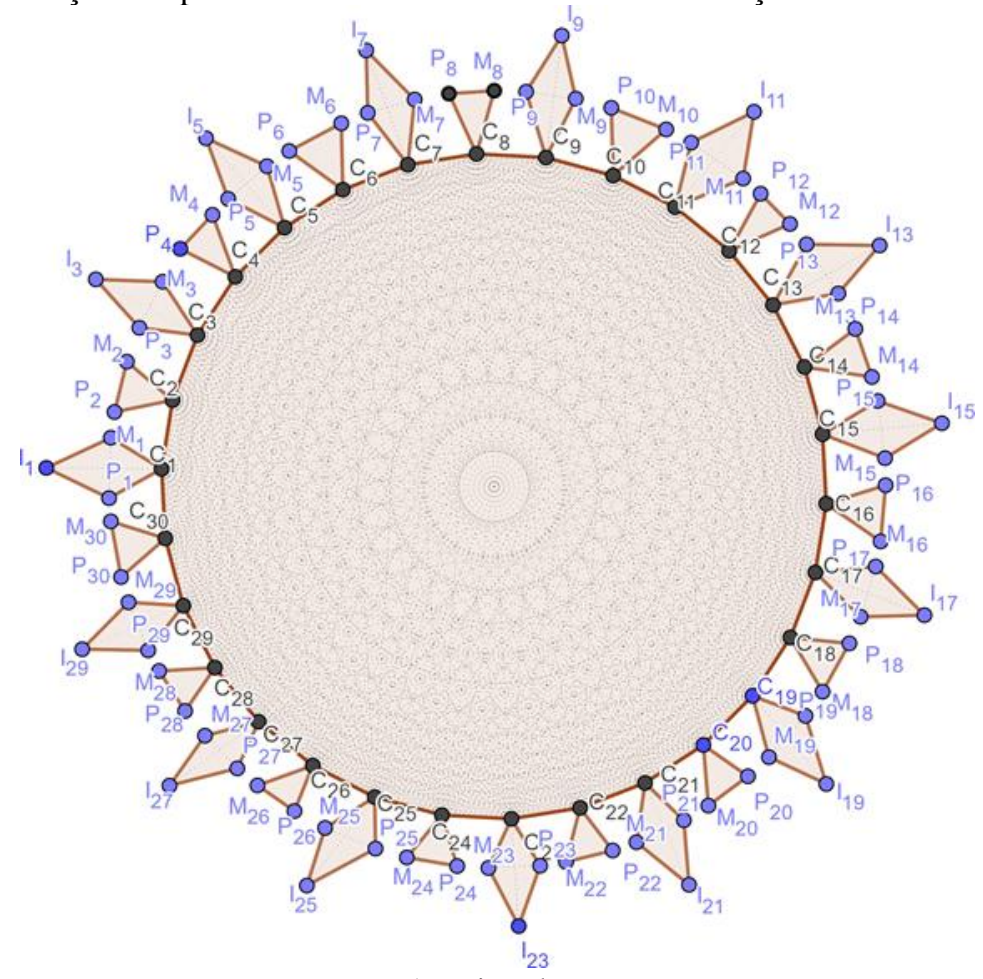

Fonte: Arquivo dos autores

Vale salientar, que nos cenários considerados não levamos em conta que os professores ministraram aulas em outras turmas com o mesmo perfil, nem que estas pessoas tiveram contato com outras pessoas fora de suas classes ou de suas residências, o que aumentaria de forma significativa 
tanto o número de pessoas expostas como o número de contatos cruzados. No cenário real, esses números são ainda maiores.

\section{CONSIDERAÇÕES FINAIS}

Diante do fato de que uma criança infectada (sintomática ou assintomática) pode transmitir o vírus para outras pessoas como colegas, professores e familiares e que as aulas presenciais provocam uma enorme movimentação entre as pessoas e consequentemente, um aumento do contato entre elas e que os estudos epidemiológicos compartimentais apontam que quanto mais contatos maior a capacidade de contágio pelo vírus SARCoV-2, a reabertura das escolas deve ser feita com muita cautela.

O risco de contágio pode ficar maior para os alunos que não manusearem de forma adequada os equipamentos de proteção individual, tais como: máscaras, protetor facial e álcool em gel, bem como para os que não compreenderem a necessidade do distanciamento social.

Com base no exposto, concluímos que o momento mais adequado para a reabertura das escolas, numa determinada população, é quando a disseminação do vírus estiver bem controlada. Isto certamente ocorre quando a maioria desta população estiver imunizada através de uma vacina ou quando os dados epidemiológicos indicarem uma imunidade coletiva. Por outro lado, se houver uma redução significativa para o número de casos e óbitos e a população (família e escola) aderir com afinco as medidas de distanciamento social e utilização de equipamentos de proteção individual poderíamos reabrir as escolas, como em outros países, sem maiores transtornos. 


\section{REFERÊNCIAS}

1. Almeida, P. R. d. et al. Modelos epidêmicos sir, contínuos e discretos, e estratégias de vacinação. Dissertação de Mestrado, Departamento de Matemática, UFV - Universidade Federal de Viçosa, Viçosa, 2014.

2. Arroyo, J., Colocar 20 crianças numa sala de aula implica em 808 contatos cruzados em dois dias, alerta universidade. Disponível em $<$ https://brasil.elpais.com/sociedade/2020-06-17/ colocar-20-criancas-numa-sala-de-aula-implica-em808-contatos-cruzados-em-dois-dias-alerta-universidade. html?ssm=whatsapp>, Acessado em $16 / 07 / 20$.

3. Batista, A. A. e Da Silva, S. H., An epidemiological compartmental model with automated parameter estimation of the spread of COVID-19 with analysis of data from Germany and Brazil. ReseachGat (preprint), 1-30 (2020). Doi:10.13140/RG.2.2.11430.75843.

4. Beghi, E., Feigin, V., Caso V., Santalucia P., Logroscino G., COVID-19 Infection and Neurological Compli- cations: Present Findings and Future Predictions. Neuroepidemiology, 1-6 2020

5. Cahapay, M. B., Asian Journal of Distance Education. Journal of Global Health, 15 no 1, 269276 (2020).

6. Castilho, C., Gondim, J. A. M. Marchesin, M., Sabeti, M., Assessing the eficiency of different control strate- gies for COVID-19 epidemic. Electronic Journal of Differential Equations, 2020 no. 64, $1-17(2020)$

7. Dong, Y., Mo, X., Hu, Y., Qi, X., Jiang, F., Jiang, Z., Tong, S., Epidemiology of COVID-19 Among Children in China. Pediatrics, 145, no 6, 1-10 (2020).

8. Farias, A. V., Um estudo da modelagem epidemiológica SIR usando conceitos de derivadas de ordem inteira e fracionária. Dissertação de Mestrado, Departamento de Matemática, UFRGS Universidade Federal do Rio Grande do Sul, Porto Alegre, 2017.

9. Fiani, B., Covarrubias, C., Desai, A., Sekhon, M., Jarrah, R., A Contemporary Review of Neurological Sequelae of COVID-19. Frontiers in Neurology, 11 no 640, 2020.

10. Kermack, W. O. e McKendrick, A. G., A Contribution to the Mathematical Theory of Epidemics. Proceedings of the Royal Society of London. Series A, Containing papers of a mathematical and physical character 115,700 (1927).

11. Kishore, J., Reopening of School in COVID 19 Pandemic. Indian Journal of Youth and Adolescent Health, 7no 1, 23-26 (2020).

12. Nicola, S. H. de J., A matemática e a epidemia. Professor de Matemática online, 8 no 3, 298306 (2020).

13. Ribeiro, S. C., Freitas, J. B., Rosa-Osman, S. M., Avaliação de fatores de risco para doenças cardiovasculares: Estudo com população de estudantes da rede pública de Manaus -AM. Latin American Journal of Development, v.2, n.6, 363-378 (2020). 
14. Riollano-Cruz, M., Akkoyun, E., Briceno-Brito, E., Kowalsky, S., Reed, J., Posada, R., Sordillo, E. M., Tosi,M., Trachtman, R., Paniz-Mondolfi, A., Multisystem inflammatory syndrome in children related to COVID-19: A New York City experience Journal of Medical Virology, 1-10 (2020).

15. Siegenfeld, C. S., Shen, C., Bar-Yam, Y., A Brief Cautionary Note on Opening Schools. New England Com-plex Systems Institute, 2020.

16. Vermund, S. H., Pitzer, V. E., Asymptomatic transmission and the infection fatality risk for COVID-19: Implications for school reopening. Clinical Infectious Diseases, (Preprint) 1-14 (2020). Doi.org/10.1093/cid/ciaa855. 